\title{
Sensitivity Based Capacitor Placement Using Cuckoo Search Algorithm for Maximum Annual Savings
}

\author{
Dinakara Prasad Reddy P, K.Gunaprasad \\ ${ }^{1}$ Lecturer, Department of EEE, Sri Venkateswara University, Tirupati, Andhra Pradesh, India. \\ ${ }^{2}$ Assistant Professor, Department of EEE, SISTK, Puttur, Andhra Pradesh, India
}

\begin{abstract}
This paper presents a two stage approach that determines the optimal location and size of capacitors on radial distribution systems to improve voltage profile and to reduce the active power loss. In first stage, the capacitor locations can be found by using loss sensitivity method. Cuckoo search algorithm is used for finding the optimal capacitor sizes in radial distribution systems. The sizes of the capacitors corresponding to maximum annual savings are determined by considering the cost of the capacitors. The proposed method is tested on 15bus, 34-bus and 69-bus test systems and the results are presented.
\end{abstract}

Keywords: - capacitor placement, loss sensitivity method, cuckoo search algorithm, radial distribution system

\section{INTRODUCTION}

In the past few decades the distribution systems were facing several persistent problems. Presently many electric companies in a number of countries experiencing very high losses. Studies shows that $13 \%$ of total power generated is wasted in the form of losses at the distribution level [1]. To reduce these losses, shunt capacitor banks are installed on radial distribution feeders. With active power loss reduction and voltage profile improvement as objectives, the optimal capacitor placement problem aims to determine the optimal capacitor location and capacitor sizes in radial distribution systems. Efficient methods are required to determine the best location and sizes. The early approaches were based on heuristic optimization algorithms. In previous methods the problem the problem are taken as a nonlinear programming model and considered both location and capacitor sizes as continuous variables [2-5].

Sundharajan and Pahwa [6] proposed the genetic algorithm approach to determine the optimal placement of capacitors. A simple heuristic numerical algorithm that is based on the method of local variation is proposed in [7]. In this paper genetic algorithm is proposed to determine the optimal selection of capacitors. Das [8] proposed the genetic algorithm approach for reactive power compensation in distribution systems to reduce the energy loss under varying load conditions. Prakash and Sydulu [9] proposed the Particle swarm optimization method to size the capacitors in distribution system capacitor placement problem. $\mathrm{Ng}$ et al [10] proposed an approach to the capacitor placement problem based on fuzzy expert system. This system containing a set of heuristic rules used to determine the capacitor placement suitability index of each node in the distribution system. Capacitors are placed on the nodes with the highest suitability index. Papers [12-14] presented a two stage methodology using Differential Evolution algorithm, Hybrid genetic algorithm and Bat algorithm for optimal capacitor sizing respectively. Branch current load flow method [11] is used in this paper.

\section{CAPACITOR LOCATIONS USING LOSS SENSITIVITY METHOD}

The loss sensitivity method is a systematic procedure of computing the maximum impact on the real power losses of the system with respect to the nodal reactive power. The relationship for computing the loss sensitivity for any bus can be derived as follows

Consider a distribution line with an impedance $\mathrm{R}+\mathrm{jX}$ and a load of Peff $+\mathrm{jQeff}$ connected between ' $\mathrm{i}$ ' and ' $\mathrm{j}$ ' buses as given below in Figure.1.

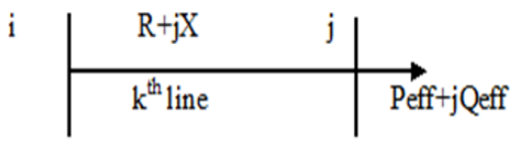

Figure 1. A distribution line with an impedance and a load.

The active power losses (PLoss) and reactive power loss (QLoss) in the distribution line are given as

$$
P_{l o s s}[j]=\frac{\left(P_{\text {eff }}^{2}[j]+Q_{e f f}^{2}[j]\right) R[k]}{(V[j])^{2}} \quad Q_{l o s s}[j]=\frac{\left(P_{e f f}^{2}[j]+Q_{e f f}^{2}[j]\right) X[k]}{(V[j])^{2}}
$$


Loss sensitivity SL, for any bus $\mathrm{j}$ can be given as $\mathrm{SLj}=\frac{\left.\left(2 * \mathrm{Q}_{\mathrm{eff}}[\mathrm{j}]\right)^{*} \mathrm{R}[\mathrm{k}]\right)}{(\mathrm{V}[\mathrm{j}])^{2}}$

Based on SLj, the buses are ranked in descending order of its values. The bus having highest numeric value is ranked top in the priority list and is considered first for capacitor placement. The buses having high value of the loss sensitivity SL, along with voltage (V) in p.u. at each bus satisfying the condition $V / 0.9>1.1$ are selected as candidate buses for capacitor placement.

Candidate location vector of 15,34 and 69 bus radial distribution system contains set of sequence of buses given as $\{6,3\},\{19,20,22\}$ and $\{57,58,61\}$ respectively.

\section{CUCKOO SEARCH (CS) ALGORITHM}

The Cuckoo search (CS) algorithm [15] is an optimization technique developed by Xin-She Yang and suash Deb in 2009. This algorithm is inspired by some species of a bird family called cuckoo because of their special life-style and aggressive reproduction strategy. These species lay their eggs in the nests and remove the existing eggs so that the hatching probability of their eggs is increased. On the other hand, some of the host birds are able to combat this parasitic behavior of cuckoos and throw out the discovered alien eggs or build their new nests in new locations.

This algorithm contains a population of nests or eggs. For simplicity, the following representations are used where each egg in a nest represents a solution and a Cuckoo egg represents a new one. If the Cuckoo egg is very similar to the host's egg, then this Cuckoo's egg is less likely to be discovered, thus the fitness should be related to the difference in solutions. The aim is to employ new and potentially better solutions (Cuckoos') to replace a not-so-good solution in the nests. For simplicity in describing the CS, the following three idealized rules are utilized:

1. Each Cuckoo lays one egg at a time, and dumps it in a randomly chosen nest.

2. The best nests with high quality of eggs are carried over to the next generations.

3. The number of available host nest is constant, and the egg which is laid by a Cuckoo is discovered by the host bird with a probability of pa in the range of $[0,1]$. The later assumption can be approximated by the fraction pa of the $n$ nests which is replaced by new ones (with new random solutions).

It is worth pointing out that, in the real world, if a cuckoo's egg is very similar to a host's eggs, then this cuckoo's egg is less likely to be discovered, thus the fitness should be related to the difference in solutions. Therefore, it is a good idea to do a random walk in a biased way with some random step sizes.

\subsection{Algorithm for Capacitor Placement and Sizing Using Loss sensitivity method and CS Algorithm}

After identifying the $\mathrm{n}$ number of candidate locations using loss sensitivity method, the capacitor sizes in all these $\mathrm{n}$ candidate locations are obtained by using the Cuckoo search algorithm. Based on the above three rules, the basic steps of the CS are framed.

Step 1: Initially [pop x $\mathrm{n}$ ] number of nest population are generated randomly within the limits Qmin and Qmax where pop is the population size and $\mathrm{n}$ is the number of capacitors

nest $(\mathrm{i},:)=\mathrm{Qmin}+(\mathrm{Qmax}-\mathrm{Qmin})$. * rand $(\operatorname{size}(\mathrm{Qmin}))$

Step 2: By placing all the $n$ capacitors of each nest at the respective candidate locations and load flow analysis is performed to find the total real power loss $\mathrm{P}_{\mathrm{L}}$. The same procedure is repeated for the nop number of particles to find the total real power losses. Fitness value corresponding to each nest is evaluated using the below equation for maximum annual savings. Fitness function for maximum savings (considering the capacitor cost) is given by

$\mathrm{S}=\mathrm{K}_{\mathrm{P}} \cdot \Delta \mathrm{P}+\mathrm{K}_{\mathrm{E}} \cdot \Delta \mathrm{E}-\mathrm{K}_{\mathrm{C}} \cdot \mathrm{Q}_{\mathrm{C}}$

Where $\mathrm{S}$ is the savings in $\$$ /year, $\mathrm{K}_{\mathrm{P}}$ is a factor to convert peak power losses to dollars, $\mathrm{K}_{\mathrm{E}}$ is a factor to convert energy losses to dollars, $\mathrm{K}_{\mathrm{C}}$ is the cost of capacitors in dollars, $\Delta \mathrm{P}$ is the reduction in peak power losses, $\Delta \mathrm{E}$ is the reduction in energy losses and $Q_{C}$ is the size of the capacitor in kvar.

The capacitor sizes corresponding to maximum savings are required. For any one nest, the negative $\mathrm{S}$ value indicates that savings are negative and $\mathrm{S}$ is fixed at $\mathrm{S}$ (minimum) and capacitor sizes corresponding to that particle are fixed at $\mathrm{Q}_{\mathrm{C}}$ (minimum).

Step 3: Start iterations

Step 4: Generate new nests using random walk (but keep the current best)

new_nest $(i,:)=$ nest + stepsize. *randn

Step 5: New fitness values are calculated for the new nests. If the new fitness value for any nest is better than previous value then pbest value for that nest is set to present fitness value. 
Step 6: A fraction of worst nests are abandoned and replace by constructing new nests with discovery rate of alien eggs (pa)

$\mathrm{k}=$ rand $($ size $($ nest $))<\mathrm{pa}$

new_nest $(\mathrm{i},:)=$ nest + stepsize. $* \mathrm{k}$

Step 7: New fitness values are calculated for the new nests. If the new fitness value for any nest is better than previous value then pbest value for that nest is set to present fitness value.

Step 8: Find the best nest so far

Step 9: The iteration count is incremented and if iteration count is not reached maximum then go to step 4.

Step 10: The capacitor sizes corresponding to maximum savings gives the optimal capacitor sizes in $\mathrm{n}$ capacitor locations and the results are printed.

\section{RESULTS}

The proposed method for loss reduction by capacitor placement is tested on IEEE 15 bus, 34 bus and 69 bus radial distribution systems. Loss sensitivity method is used to find the optimal capacitor locations and CS algorithm is used to find the optimal capacitor sizes for maximum annual savings. The data used for finding the optimal capacitor sizes are nop $=50, \mathrm{Qmin}=100 \mathrm{kvar}$, Qmax=1500 kvar, pa=0.25, $\mathrm{K}_{\mathrm{P}}=150 \$ / \mathrm{kW}, \mathrm{K}_{\mathrm{E}}=0.06$ $\$ / \mathrm{kWh}, \mathrm{K}_{\mathrm{C}}=5 \$ / \mathrm{kVAr}$ and Itmax $=1000$.

Table 1. Results for 15 Bus System

\begin{tabular}{|c|c|}
\hline Bus No & Size (kvar) \\
\hline 3 & 693 \\
\hline 6 & 334 \\
\hline Total kVAr placed & 1027 \\
\hline Total Power loss in kW (before) & 61.7944 \\
\hline Total Power loss in kW (after) & 33.3302 \\
\hline Savings in dollars & $\$ 14,012$ \\
\hline
\end{tabular}

Table 2. Results for 34 Bus System

\begin{tabular}{|c|c|}
\hline Bus No & Size(kvar) \\
\hline 19 & 623 \\
\hline 22 & 861 \\
\hline 20 & 229 \\
\hline Total kvar placed & 1713 \\
\hline Total Power loss in kW (before) & 221.7235 \\
\hline Total Power loss in kW (after) & 170.0478 \\
\hline Savings in dollars & $\$ 26,182$ \\
\hline
\end{tabular}

Table 3. Results for 69 Bus System

\begin{tabular}{|c|c|}
\hline Bus No & Size(Kvar) \\
\hline 57 & 74 \\
\hline 58 & 66 \\
\hline 61 & 1142 \\
\hline Total kvar placed & 1282 \\
\hline Total Power loss in kW & 225 \\
\hline Total Power loss in $\mathrm{kW}$ & 151.9464 \\
\hline Savings in dollars & $\$ 42,737$ \\
\hline
\end{tabular}

\section{CONCLUSION}

In this paper, a two stage methodology of finding the optimal locations and sizes of shunt capacitors for reactive power compensation of radial distribution systems is presented. Loss sensitivity method is proposed to find the optimal capacitor locations and cuckoo search algorithm is proposed to find the optimal capacitor sizes. By installing shunt capacitors at all the potential locations, the total real power loss of the system has been reduced significantly and at same time annual savings are increased and bus voltages are improved substantially. The proposed method is tested on IEEE 15, 34 and 69 bus radial distribution systems. The proposed CS method 
iteratively searches the optimal capacitor sizes for the maximum annual savings. CS algorithm is less complex because less parameters are there when compared to other algorithms.

\section{REFERENCES}

[1] H. N. Ng, M. M. A. Salama, and A.Y. Chikhani "Classification of capacitor allocation techniques" IEEE Trans. Power Delivery, vol.15, pp387-392, Jan.2000.

[2] Duran H., "Optimum number, location and size of shunt capacitors in radial distribution feeders: A dynamic programming approach", IEEE Transactions on Power Apparatus and Systems, vol. - 87, no. 9, pp. 1769-1774, September 1968.

[3] Grainger J.J and S.H. Lee, "Optimum size and location of shunt capacitors for reduction losses on distribution feeders" IEEE Transactions on Power Apparatus and Systems, vol. PAS- 100, no. 3, pp. 1105-1118, March 1981.

[4] Baran M.E. and Wu F.F., "Optimal capacitor placement on radial distribution systems",IEEE Transactions on Power Delivery, vol.4,no-1, pp. 725-734, January 1989.

[5] Baran M.E. and Wu F.F., "Optimal sizing of capacitors placed on a radial distribution system", IEEE Transactions on Power Delivery, vol.4, no-1, pp. 735-743, January 1989.

[6] Sundhararajan S. and Pahwa A., "Optimal selection of capacitors for radial distribution systems using a genetic algorithm", IEEETransactions on Power Systems, vo1.9, no.3, pp.1499-1507, August 1994.

[7] Chis M., Salama M.M.A. and Jayaram "Capacitor placement in distribution systems using heuristic search strategies", IEE proceedings on Generation, Transmission and Distribution, vol. 144, no.3, pp. 225-230, May 1997.

[8] D. Das, "Reactive power compensation for radial distribution networks using geneticalgorithms," Electric Power and Energy Systems, Vol. 24, 2002, pp.573-581.

[9] Prakash K. and Sydulu M., "Particle swarm optimization based capacitor placement on radial distribution systems", IEEE Power Engineering Society general meeting 2007, pp. 1-5, June 2007.

[10] Ng H.N., Salama M.M.A. and Chikhani .Y., "Capacitor allocation by approximate reasoning: fuzzy capacitor placement", IEEE Transactions on Power Delivery, vol. 15, no.1, pp 393 - 398, January 2000.

[11] Das D., Kothari D.P. and Kalam A., "Simple and efficient method for load flow solution of radial distribution networks", Electrical Power \& Energy Systems, vol. 17, no. 5, pp.335-346, 1995.

[12] V. Usha Reddy, Dr .T. Gowri Manohar, P.Dinakara Prasad Reddy, "Capacitor Placement for loss reduction in radial distribution networks: A two stage approach", Journal of electrical engineering, Volume 12, edition 2, 2012.

[13] Dinakara Prasad Reddy P, Sreenivasulu A . " Optimal Capacitor Placement for Loss Reduction in Distribution Systems Using Fuzzy and Hybrid Genetic Algorithm ", Vol.2 - Issue 11 (November - 2013), International Journal of Engineering Research \& Technology (IJERT), ISSN: 2278-0181, www.ijert.org

[14] Mrs V. USHA REDDY, Dr T. GOWRI MANOHAR and P.DINAKARA PRASAD REDDY, 2013. OPTIMAL CAPACITOR PLACEMENT FOR LOSS REDUCTION IN DISTRIBUTION SYSTEMS BY USING BAT ALGORITHM .International Journal of Electrical Engineering \& Technology (IJEET).Volume:4,Issue:2,Pages:338-343.

[15] X.S. Yang, S. Deb, Cuckoo search via Levy flights, World Congress on Nature \& Biologically Inspired Computing (NaBIC 2009), December 2009, India, IEEE Publications, USA, pp. 210-214 (2009). 